\title{
The Dark Side of Gefitinib: Reflectance Confocal Microscopy Applied to Hair Hyperpigmentation
}

\author{
Terenzio Cosio Sara Mazzilli Luca Bianchi Elena Campione \\ Dermatologic Unit, Department of Systems Medicine, University of Rome Tor Vergata, Rome, Italy
}

\section{Established Facts}

- Already known facts: patients treated with gefitinib could suffer from the PRIDE complex, which implies also hair and eyebrow depigmentation.

- Already known facts: neoplastic cells can develop resistance to gefitinib by reactivating the oct4 pathway. Even other pathways could be altered in the stem niche and not only in a tumor environment.

\section{Novel Insights}

- This is the first witnessed case in which a therapy with epidermal growth factor receptor inhibitors leads to hyperpigmentation in hair and eyebrows.

- Systemic chemotherapy may activate survival pathways in senescent cells due to polymorphisms.

\section{Keywords}

Hair hyperpigmentation · oct4 · Gefitinib · PRIDE complex · Reflectance confocal microscopy

\begin{abstract}
Gefitinib is a multi-target tyrosine kinase inhibitor used for the treatment of non-small cell lung cancer. Papulo-pustular and/or paronychia, abnormalities in hair growth, itching, and dryness due to epidermal growth factor inhibitors - i.e., PRIDE Complex - are a common effect of tyrosine kinase inhibitors. We report a case of hair and eyebrow hyperpigmentation after 7 months of treatment with gefitinib. In the literature, we found no data regarding rapid pigmentation of hair due to treatment with any multi-target tyrosine kinase inhibitors. To our knowledge, this is the first case reporting
\end{abstract}

both hair and eyebrow hyperpigmentation. We hypothesize the role of different mechanisms linked to rapid hair hyperpigmentation.

(c) 2019 S. Karger AG, Basel

\section{Introduction}

Gefitinib is a multi-target tyrosine kinase inhibitor used for the treatment of non-small cell lung cancer (NSCLC).

Papulo-pustular and/or paronychia, abnormalities in hair growth, itching, and dryness due to epidermal growth factor inhibitors - i.e., PRIDE complex - are a common effect of tyrosine kinase inhibitors [1].

In the literature, no data regarding rapid pigmentation of hair due to treatment with any multi-target tyrosine 
Fig. 1. a Photograph from June 9, 2017, showing the patient with gray hair. b Photograph from May 3, 2019, showing the patient with black hair and eyebrows.
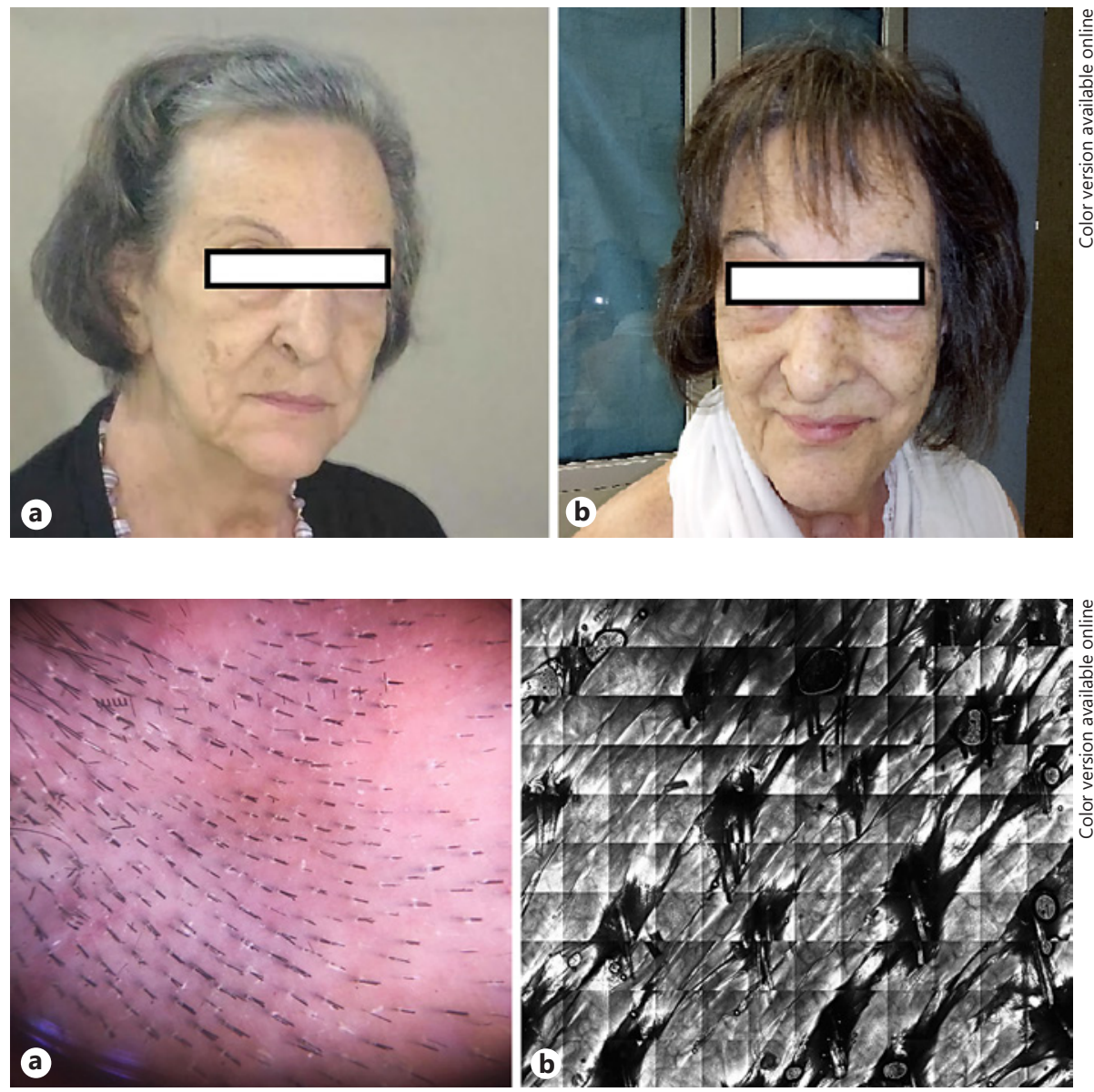

Fig. 2. a Dermoscopy of the patient's occipital region of the scalp showing brown scalp hyper-pigmentation, absence of erythema and vessels, hair bigeminy, and non-homogeneous hair bulb distribution. b RCM: the mosaic describes hair bulb bigeminy associated with the presence of pigment around the follicles. kinase inhibitors are reported. We hypothesize the role of different mechanisms linked to rapid hair hyperpigmentation.

\section{Case Report}

A 77-year-old woman was visited in our clinic for dorsal large basal cell carcinoma. She also reported a strong unexpected hair regrowth and re-pigmentation in the last 7 months not correlated to any vitamin supplement during treatment with gefitinib for (NSCLC) (Fig. 1).

Hair hypertrichosis linked to gefitinib is reported in the literature as eyelash hypertrichosis [2], while there are contrasting reports of cutaneous hyperpigmentation and vitiligo [3]. There are no reports in the literature regarding rapid variations in the color of eyebrows, eyelashes, or other hairy area of the body. Dermoscopy showed brown scalp hyperpigmentation, absence of erythema and vessels, hair bigeminy, and non-homogeneous hair bulb distribution (Fig. 2a).

We performed reflectance confocal microscopy (RCM) to evaluate morphological changes in the hair follicle and perifollicular area (Fig. 2b). The mosaic describes hair bulb bigeminy/trigeminy and pigmented keratinocytes that have an epidermal honeycomb pattern with interfollicular hyperreflective structures.

\section{Discussion}

Epidermal growth factor receptor (EGFR) has an essential role in the regulation, proliferation, and differentiation in human skin, even in basal epidermal keratinocytes and hair follicles [4-6]. All previous studies on EGFR demonstrate alteration in hair growth and color in human beings and mice [7]: the EGFR-ligand system in the hair follicle has an essential role in regulating the hair cycle, as stimulating transition from anagen to catagen of EGFR activation [8]. Mice with a targeted disruption of the EGFR allele have short and wavy hair that becomes progressively atrophic leading to alopecia $[7,9]$. The hair follicles in these mice do not develop from the anagen to the telogen phase and are destroyed by inflammatory infiltration. Even in our case - opposite to what is indicated in the data - we identified no local inflammatory infiltration, no damage to the follicle and, actually, due to the presence of bigeminy, an index of follicular reactivation. This aspect was confirmed by RCM performed after 7 months of treatment with gefitinib, which described 
hair bulb bigeminy and pigmented keratinocytes that have an epidermal honeycomb pattern with interfollicular hyperreflective structures. Dermoscopy showed brown scalp hyperpigmentation, absence of erythema and vessels, pili bigeminy, and non-homogeneous hair bulb distribution. Skin hyperpigmentation could be related to phagocytized melanin macrophage in the superficial dermis [10].

Philpott et al. [7] reported that EGF may induce an artificial 'catagen-like' effect by stimulating hair outer sheath growth, interrupting the normal patterns of proliferation and migration that occur in the anagen hair follicle responsible of an anagen-to-catagen-like transition [11]. Thus, the use of an EGFR inhibitor may block the anagen-to-catagen switch.

We investigated even other markers; hair pigmentation is regulated by several factors including stem cell factor (SCF) and its receptor c-kit (CD117) whose normal interaction is required for preserving hair follicle melanocytes [12]. SCF/c-kit signaling pathway facilitates the generation and migration of functional melanocytes during each new hair cycle. This has been shown in different populations of the hair follicle melanocytes, expressing a different dependence on SCF during cyclic regeneration of the hair pigmentary unit. Gene mutation has been described in the literature in case of hair depigmentation [13], but not in hair hyperpigmentation. Therefore, based on literature review, we supposed that hypertrichosis and hyperpigmentation could be driven by oct 4 (octamer-binding transcription factor 4) [14] and c-kit mutation in the follicle niche, via CD-133 [15, 16] parallel to the migration of melanoblasts in the anagen phase [17].

Follicular melanocytes, keratinocytes, and dermal papilla cells have mutual interactions in hair bulbs. Dermal papilla give signals to melanocytes, transferring melanin granules into the keratinocytes. Perturbations of these interactions could affect the amount and type of melanin delivered to the hair [18]. MC1R (melanocortin 1 receptor) is a G-protein-coupled receptor expressed on the surface of skin and hair melanocytes. The binding of the MC1R ligand - $a$-melanocyte-stimulating hormone $(\alpha-\mathrm{MSH})$ - induces a melanogenic cascade, leading to the interruption of dark eumelanin production. Eumelanin synthesizes phaeomelanin, a red or yellow pigment. In many vertebrate species, the loss of MC1R signaling stops melanocytes from producing eumelanin [18]. As a Gprotein, $\mathrm{MC} 1 \mathrm{R}$ is also involved in pigment reproduction. Thus, we thought it could be interesting to follow a mechanism of resistance induced by gefitinib. Melanocytes re- spond to the gefitinib stimulation expressing or reactivating isoforms of MC1R, which, together with CD-133, leads to white hair pigmentation.

However, an unknown polymorphism may influence this unique phenotype. Therefore, in the future, it may be necessary to have a pharmacogenomic evaluation on offlabel targets and above all on skin appendages.

\section{Conclusion}

This case highlights a pleomorphic individual response to systemic anti-neoplastic therapies, underling the genotypic support for each therapeutic option. The use of dermoscopy and RCM in therapeutic monitoring of skin hyperpigmentation during treatment with gefitinib could be a useful and non-invasive diagnostic monitoring tool to evaluate skin hyperpigmentation. Nonetheless, future studies are required to evaluate the change at the bulbar stem cells level for systemic treatments.

\section{Acknowledgement}

We thank Prof. Denis Mariano for his contribution in editing the study.

\section{Statement of Ethics}

The patient has given her consent to publish details and photos of the case.

\section{Disclosure Statement}

The authors have no conflicts of interest to declare.

\section{Funding Sources}

No funding sources have been used.

\section{Author Contributions}

The authors have contributed equally to the drafting of the paper. Each author has (1) made substantial contributions to the conception or design of the work, or to the acquisition, analysis, or interpretation of data for the work; (2) participated in drafting the work or revising it critically for important intellectual content; (3) approved the final version to be published; and (4) agreed to be accountable for all aspects of the work in ensuring that questions related to the accuracy or integrity of any part of the work are appropriately investigated and resolved.
Cosio/Mazzilli/Bianchi/Campione 


\section{References}

1 Madke B, Gole P, Kumar P, Khopkar U. Dermatological Side Effects of Epidermal Growth Factor Receptor Inhibitors: 'PRIDE' Complex. Indian J Dermatol. 2014 May;59(3):2714

2 Agrawal CR, Goyal P, Doval DC, Dutta K, Domadia K, Kothiwal S. Eyelashes Trichomegaly: An Unusual Side Effect of Gefitinib Therapy. Int J Trichology. 2018 Jan-Feb; 10(1):44-6.

3 Sheila Z. Jalalat BS1 and Philip R. Cohen. Gefitinib-associated vitiligo: report in a man with parotid squamous cell carcinoma and review of drug-induced hypopigmentation. Dermatol Online J. 2013 Oct;19(10): 20020.

4 Nanney LB, Magid M, Stoscheck CM, et al: Comparison of epidermal growth factor binding and receptor distribution in normal human epidermis and epidermal appendages. J Invest Dermatol. 1984 Nov;83(5):38593.

5 Jost M, Kari C, Rodeck U: The EGF receptor - an essential regulator of multiple epidermal functions. Eur J Dermatol. 2000 Oct-Nov; 10(7):505-10.

6 Van Doorn R, Kirtschig G, Scheffer E, Stoof TJ, Giaccone G. Follicular and epidermal alterations in patients treated with ZD1839 (Iressa), an inhibitor of the epidermal growth factor receptor. Br J Dermatol. 2002 Sep; 147(3):598-601.
7 Philpott MP, Kealey T. Effects of EGF on the morphology and patterns of DNA synthesis in isolated human hair follicles. J Invest Dermatol. 1994 Feb;102(2):186-91.

8 Murillas R, Larcher F, Conti CJ, Santos M, Ullrich A, Jorcano JL. Expression of a dominant negative mutant of epidermal growth factor receptor in the epidermis of transgenic mice elicits striking alterations in hair follicle development and skin structure. EMBO J. 1995 Nov; 14(21):5216-23.

9 Hansen LA, Alexander N, Hogan ME, Sundberg JP, Dlugosz A, Threadgill DW, et al. Genetically null mice reveal a central role for epidermal growth factor receptor in the differentiation of the hair follicle and normal hair development. Am J Pathol. 1997 Jun;150(6): 1959-75.

10 Chang GC, Yang TY, Chen KC, Yin MC, Wang RC, Lin YC. Complications of therapy in cancer patients: Case 1. Paronychia and skin hyperpigmentation induced by gefitinib in advanced non-small-cell lung cancer. J Clin Oncol. 2004 Nov;22(22):4646-8

11 Schallreuter K, Slominski A, Pawelek JM, Jimbow K, Gilchrest BA. What controls melanogenesis? Exp Dermatol. 1998 Aug;7(4):14350.

12 Botchkareva NV, Khlgatian M, Longley BJ, Botchkarev VA, Gilchrest BA. SCF/c-kit signaling is required for cyclic regeneration of the hair pigmentation unit. FASEB J. 2001 Mar;15(3):645-58.
13 Villodre ES, Kipper FC, Pereira MB, Lenz G. Roles of OCT4 in tumorigenesis, cancer therapy resistance and prognosis. Cancer Treat Rev. 2016 Dec;51:1-9.

14 Kobayashi I, Takahashi F, Nurwidya F, Nara T, Hashimoto M, Murakami A, Yagishita S, Tajima K, Hidayat M, Shimada N, Suina K, Yoshioka Y, Sasaki S, Moriyama M, Moriyama H, Takahashi K. Oct4 plays a crucial role in the maintenance of gefitinib-resistant lung cancer stem cells. Biochem Biophys Res Commun. 2016 Apr;473(1):125-32.

15 Zhou L, Xu M, Yang Y, Yang K, Wickett RR, Andl T, et al. Activation of $\beta$-Catenin Signaling in CD133-Positive Dermal Papilla Cells Drives Postnatal Hair Growth. PLoS One. 2016 Jul;11(7):e0160425.

16 Grichnik JM, Burch JA, Burchette J, Shea CR. The SCF/KIT pathway plays a critical role in the control of normal human melanocyte homeostasis. J Invest Dermatol. 1998 Aug; 111(2):233-8.

17 Morgan MD, Pairo-Castineira E, Rawlik K, et al. Genome-wide study of hair colour in UK Biobank explains most of the SNP heritability. Nat Commun. 2018 Dec;9(1):5271. https:// doi.org/10.1038/s41467-018-07691-z.

18 Wolf Horrell EM, Boulanger MC, D'Orazio JA. Melanocortin 1 Receptor: Structure, Function, and Regulation. Front Genet. 2016 May;7:95.
Hair and Eyebrow Hyperpigmentation Induced by Gefitinib in Advanced NSCLC 